\section{The pathogenesis of prostate cancer: from molecular to metabolic alterations}

\author{
Elisa Benedettini
}

Paul Nguyen

Massimo Loda

\begin{abstract}
Prostate cancer $(\mathrm{PCa})$ is a heterogeneous disease with regard to molecular alterations and clinical course. The investigation of genetic alterations associated with PCa pathogenesis is highly challenging. Genome-wide analyses and epidemiological studies have identified only a handful of candidate genes possibly associated with hereditary or sporadic PCa. Cancer cells often rely for survival on common biochemical pathways such as enhanced anaerobic glycolysis and lipogenesis. The lipogenic enzyme fatty acid synthase seems to play a crucial part in PCa by conferring growth and survival advantages to cancer cells. We summarize the current understanding of the molecular events in PCa, and highlight the importance of altered lipid metabolism in the development and progression of prostate malignancy.
\end{abstract}

Keywords FASN; genetic mutations; lipid metabolism; prostate cancer; risk stratification; therapeutic approaches

\section{The clinical problem}

\section{Epidemiology}

Among the male population of the USA, prostate cancer is the most prevalent non-cutaneous neoplasm ( 1 in 6 men will be diagnosed with PCa cancer during their lifetime) and a leading cause of cancer-related death, second only to lung and colon cancer. ${ }^{1}$ Its frequency varies widely, with the highest rates reported in Western countries, and the lowest rates in Asia. ${ }^{2}$ Multiple factors contribute to the high incidence and prevalence of PCa. Risk factors include age, family history and race, but

Elisa Benedettini PhD is a Postdoctoral Fellow at Dana-Farber Cancer Institute, Massachusetts, USA.

Paul Nguyen мD is a Resident in Radiation Oncology at Dana-Farber Cancer Institute and Harvard Medical School Massachusetts, USA.

Massimo Loda MD is a Professor of Pathology at Department of Pathology, Dana-Farber Cancer Institute, Brigham and Woman's Hospital and Harvard Medical School Massachusetts, USA. He is the Director of the Center for Molecular Oncologic Pathology, Dana Farber Cancer Institute. also a diet high in fat, and obesity. Higher body mass index (BMI) and adult weight gain increase the risk of dying from prostate cancer. ${ }^{3}$

\section{Stage migration and risk stratification}

The early 1990s saw a dramatic increase in the incidence of prostate cancer with the introduction of the prostate-specific antigen (PSA) blood screening test. As screening became more widespread in the USA, there was a significant stage migration toward detection of prostate cancer at earlier stages. For example, results at the time of diagnosis dropped from $16 \%$ in the 1980 s to $4 \%$ in $2003 .{ }^{4}$ In 2004, results from the Prostate Cancer Prevention trial showed that even men with PSA less than the traditional cut-off of $4.0 \mathrm{ng} / \mathrm{ml}$ had a substantial risk of harbouring prostate cancer $(15.2 \%$ for all patients with PSA $<4.0 ; 26.9 \%$ for patients with PSA 3.1-4.0), which led many clinicians to use variations in PSA measurement as an indication to biopsy of men with PSA $<4.0$, thereby further augmenting the continued stage migration effect. ${ }^{5}$

Among men with clinically localized prostate cancer, treatment decisions of whether to pursue a local-only therapy (e.g. surgery, radiation, brachytherapy) versus a more aggressive combined method therapy (radiation plus hormones) is typically made based on the risk of recurrence. The commonest stratification system was developed by D'Amico et al. and uses clinical T-stage, PSA, and Gleason score to divide patients into low, intermediate, and high risk. ${ }^{6}$ Due to stage migration, the proportion of people with low-risk disease increased from 30\% in 1990 to $45 \%$ in 2000, and it is likely that low-risk disease represents most diagnoses of prostate cancer. ${ }^{7}$

For patients with low-risk disease, it is likely that a large proportion do not need treatment. The risk stratification system is not good enough to discern, based on clinical and pathological parameters, which low-risk patients have disease that will never progress from those who will die from their disease if left untreated (even though these represent the minority of low-risk patients). The net result is that many patients who do not need treatment may choose to be treated and suffer side effects unnecessarily, while others who need treatment may forgo therapy and miss a crucial opportunity to be cured. There is therefore a significant need for an improved risk-stratification system that incorporates novel biomarkers from relevant pathways to better determine the risk of recurrence and allow patients to make more informed decisions about treatment. Patients who recur and then develop metastases may be treated with androgen deprivation therapy for a while, but nearly all will eventually develop androgen-independent disease if they do not die of other causes. Median survival in the androgen-independent state is only $\sim 18$ months, and few treatment options are available which have shown benefit at this stage besides docetaxel, which extends life expectancy by only $\sim 2-3$ months over the prior standard of care. ${ }^{8}$ There is an equally urgent need to investigate relevant pathways in prostate cancer for new biomarkers and risk stratification, and for the development of novel agents that can prolong survival.

This review discusses genetic markers and molecular pathways in prostate cancer with a particular emphasis on fatty acid synthase (FASN) and its possible role as a biomarker and therapeutic target. 


\section{Genetic mutations and epigenetic changes in prostate cancer}

Analyses of polymorphic microsatellites have shown that multiple foci of cancer arise independently within the same prostate. ${ }^{9}$ An increasing number of studies prove that prostate cancer can be genetically classified into different subgroups, exemplifying the extensive phenotypic and molecular heterogeneity of this disease. Genetic profile analyses have detected only a few, potentially significant mutations in hereditary and sporadic prostate cancer.

\section{Hereditary PCa}

Difficulties in identifying genes that are highly penetrant in PCa can be attributed to:

- the advanced age of patients at PCa onset (median age: 60 years) makes the collection and molecular analyses of samples from more than two generations almost impossible

- the high frequency of PCa makes it difficult to distinguish sporadic cases from hereditary cases in families with high rates of disease

- hereditary PCas do not have specific clinical (except perhaps an earlier age of onset) or pathological characteristics that differentiate them from sporadic cases.

Several studies have shown a familial predisposition for PCa. Over the last two decades, genome-wide analyses and genetic epidemiologic studies of PCa have led to the identification of high-risk alleles at several genetic loci. ${ }^{10}$ However among these, only a few candidate genes may be promising, in particular RNAseL and MSR.

RNAse L expresses an endoribonuclease related to the antiviral and pro-apoptotic interferon-induced activities and maps to the HPC1 locus at 1q24-25. MSR1 encodes for a macrophage-specific receptor that binds polyanionic ligands (including bacteria, oxidized serum lipoproteins, apoptotic cells) and is located on 8p2223. Alterations in these two genes appear to be associated with progression and severity of PCa. Unlike breast cancer, for which familiar predisposition is strongly linked to polymorphisms in the genes BCRA 1 and BCRA 2, alterations in the RNAse L and/ or MSR1 genes do not account for most hereditary PCa cases. Freedman et al., using a whole-genome single nucleotide polymorphism analysis (SNP), identified a new locus at $8 \mathrm{q} 24$ that increases the risk for PCa in African-Americans and is associated with early onset of the malignancy. ${ }^{11}$ They also showed that the alleles previously identified in this locus ${ }^{12}$ are insufficient to explain the frequency of $8 \mathrm{q} 24$ amplification, pointing out that unmapped risk alleles in this region are yet to be identified. This line of investigation appears to hold the most promise to shed light on the pathogenesis of hereditary PCa.

\section{Sporadic PCa}

Sporadic PCa often shows heterogeneous patterns of oncogene activation, and is rarely associated with mutations in classic oncogenes or tumour suppressor genes. For these reasons the investigation of oncogene expression profile correlated to disease development and progression is highly challenging. Recently, genome-wide tools (e.g. comparative genome hybridization, spectral karyotyping, SNP analysis) have provided insight into common PCa chromosomal alterations ${ }^{13}$ As for hereditary PCa, only a handful of candidate genes have shown promise in having a causative role. ${ }^{14}$ These genes can be distinguished into those playing an active part in the early and those in the late phases of carcinogenesis. Genes having a putative role in tumour initiation encode for:

- the tumour suppressor proteins Pten, p27, Nkx3.1and Rb

- the transcription factor Myc

- glutathione S-transferase- $\pi$ (GSTP1) which has a role on damage-related stress prevention

- hepsin (cell-surface serine protease)

- Alpha-methylacyl-CoA racemase (AMACR), an enzyme involved in $\beta$-oxidation of branched-chain fatty acids.

The genes aforesaid have been found to be overexpressed in most gene expression profiling studies. ${ }^{15} \mathrm{KLF} 6$, a zinc finger transcription factor, the polycomb protein EZH2 (enhancer of zeste homolog 2) and telomerase have also been implicated as well in the early phases of prostate carcinogenesis.

In contrast, genes involved in cancer progression and metastases include the androgen receptor (AR) p53, Bcl2, ETV1 and ERG1. The role in tumour development and progression for most of the genes mentioned above has been supported by relevant mouse models generated in the last two decades, which often mimic many of the characteristics of the human cancer. ${ }^{16}$ Most models result in focal areas of prostatic intraepithelial neoplasia (PIN), but not in infiltrating tumours, ${ }^{17}$ suggesting that individual aberrant activation/deletion of these genes is not sufficient to produce invasive PCa and may require other secondary events for cancer progression. Other models rapidly progress to local invasive adenocarcinoma followed by metastasis, as c-myc transgenic mice, prostate conditional Pten knockout, and the compound mice Pten $+/-$; p27 null, and p53- $\underline{\mathrm{Rb}}$ - conditional knock-out mice. ${ }^{16}$

Along with mutations in individual genes, a chromosomal translocation has recently been identified in $>50 \%$ of PCa. By using a bioinformatics approach able to discover candidate oncogenic chromosomal aberrations on the basis of outlier gene expression (COPA), strong and mutually exclusive outlier profiles for ERG and ETV1 (both members of ETS transcription factor family) were identified in PCa patients ${ }^{18}$ This imbalance resulted from the fusion of TMPRSS2 (21q22.2), coding for an androgenregulated transmembrane serine protease, with ERG (21q22.3) or ETV1 (7p21.2) (and rarely with other members of the ETS family) and represents the commonest rearrangement identified in human solid tumours. As a consequence, ERG and ETV1 oncogenes are regulated by the androgen-responsive promoter of TMPRSS2, possibly providing a selective mechanism for early transformation. The occurrence of TMPRSS2:ERG or TMPRSS2: ETV1 translocations appears to be mutually exclusive, suggesting a specific mechanism required for tumour initiation or, more likely, maintenance. The TMPRSS2:ERG rearrangement, the commonest, is rather complex because both genes are located on the same chromosome and have the same transcriptional orientation. The translocation can generate 8 different types of mRNA, by alternative splicing and variant breakpoints. ${ }^{19}$ One of these mRNAs, known as TMPRSS2 ATG (with the translation initiation codon ATG of TMPRSS2 in frame with the exon 4 of ERG protein), seems to be associated with aggressive disease. ${ }^{19}$

Over the last decade, PCa has also been investigated for epigenetic changes-inheritable gene expression alterations 
without alterations in DNA sequence-which are proving to be of relevance. Studies on DNA methylation status have shown hypermethylation of $\mathrm{CpG}$ islands in the promoter of several tumour suppressor genes-e.g. Rb, p16 $6^{\mathrm{INK} 4 \mathrm{~A}}$, MLH1, MSH2, GSTP1 and APC1 — which are thereby transcriptionally silenced. ${ }^{20}$ Most promising among many potential oncogenetic candidates is GSTP1, a member of a family of enzymes playing an important part in cell detoxification. Methylation of GSTP1 is detected in several cancer types, including breast and hepatocellular carcinomas, but only in PCa does it have a prevalence of $>90 \%$ (reviewed $i^{21}$ ). This alteration has also been detected with high frequency in proliferative inflammatory atrophy (PIA). PIA is proposed to be a common proliferative response to environmental damage and, because it may be seen in proximity to high-grade prostatic intraepithelial neoplasia (HGPIN) and expresses molecular signals of early neoplastic transformation (that is, GSTP1 hypermethylation) it has been suggested that PIA is an early precursor of PCa (reviewed in ${ }^{21}$ ).

Recently, GSTP1 methylation analysis has been detected in cell-free circulating DNA, which has turned out to be a promising seric biomarker of PCa detection and possibly biochemical recurrence. $^{22}$

\section{Metabolic alterations in tumour initiation and in androgen-independent disease}

Pioneering efforts on the characterization of tumour metabolism have revealed that cancer cells rely on anaerobic pathways to convert glucose to ATP, even in abundant oxygen. ${ }^{23}$ This phenomenon, known as the Warburg effect, occurs despite the fact that the anaerobic pathway is less efficient for energy supply than aerobic respiration.

Warburg claimed that the first phase of cancer genesis is irreversible injury to the respiratory chain. Due to the resulting defective aerobic energy prodution, injured cells switch to fermentation (glycolysis with lactate as the final product) to survive. ${ }^{23}$ If on the one hand glycolysis is less efficient than aerobic respiration, on the other hand it produces ATP at a rate 100-times faster than mitochondrial oxidation. Tumour cells therefore efficiently extract glucose from the host and process it by adaptive metabolic activities. The high intake of glucose provides, by pyruvate synthesis, energy and a readily available source of acetyl CoA for mitochondrial synthesis of citrate, necessary for fatty acid/cholesterol synthesis (Figure 1). ${ }^{24}$ Under normal conditions, most fatty acids are derived from intake in food, and their endogenous synthesis is usually minimal while, in tumour cells, almost all fatty acids are produced via de novo synthesis, despite high levels of ambient fatty acids. ${ }^{25}$ Thus it is speculated that the increased metabolism of glucose contributes to the tumorigenic process by promoting de novo synthesis of fatty acids, ${ }^{26}$ even if the mechanism by which fatty acids might promote prostate cancer growth and survival is poorly understood. Consistent with this hypothesis, numerous studies show that most lipogenic enzymes, such as fatty acid synthase (FASN) and acetyl-CoA carboxylase, are overexpressed in a wide variety of tumour types. The overexpression of FASN in the prostate occurs during the earliest stages of neoplastic transformation (PIN lesions) and in nearly all invasive prostate carcinomas. ${ }^{21,25}$ Altered metabolism is not a cause of malignancies, although tumour cells may depend on altered metabolic pathways (see below).

Next, we review the evidence supporting a crucial role of fatty acid-related metabolism in the pathogenesis and progression of prostate malignancy, pointing out that the understanding of the alterations in intermediary metabolism of prostate cells may offer new opportunities in the prevention, diagnosis and treatment of PCa.

\section{FASN as a metabolic oncogene in PCa}

FASN, a $270 \mathrm{kD}$ cytosolic complex enzyme that functions as a homodimer, catalyzes the synthesis of palmitate by the condensation of malonyl-CoA and acetyl-CoA. FASN plays an important part in energy homeostasis by converting excess carbon intake into fatty acids for storage. For the reason mentioned above, FASN is expressed at low-to-undetectable levels in normal adult human tissues, except in the liver, adipose tissue, and breast of lactating women. FASN is overexpressed in many human cancers, including $\mathrm{PCa}^{27}$ We have previously shown that FASN expression progressively increases throughout the natural history of a substantial proportion (two-thirds) of PCas, beginning with PIN, and reaching the highest values in androgen-independent PCas metastatic to bone. FASN-overexpressing PCas have a distinctive gene expression signature. ${ }^{28}$

Human PCas have genomic amplification of FASN (reviewed $i^{26}$ ), suggesting that FASN confers a selective growth advantage to tumour cells. The biochemical and metabolic basis for this overexpression and its consequences are not well understood, but it is known that prostate tumours expressing high FASN levels are associated with poor prognosis (rev in ${ }^{29}$ ).

Treatment of human PCa cell lines with cerulenin, a natural irreversible inhibitor of FASN, reduces cell proliferation and, more significantly, induces cell death, ${ }^{26,30}$ while systemic treatment of nude mice bearing PCa xenografts with the more stable fatty acid synthase inhibitor C75 ( $\alpha$-methylene- $\gamma$-butyrolactone) significantly reduces the size of PCa masses that overexpress FASN. ${ }^{30}$ A recent study has shown that FASN expression and activity is higher in TRAMP mouse models compared to nontransgenic littermates, and increases with age, tumour progression, and in metastatic lesions. In these models, FASN activity inhibited by cerulenin and C-75 results in apoptosis. ${ }^{31}$

Polyphenol epigallocatecin gallate (EGCG), in part responsible for the cancer-preventive effects of green tea, is also a potent inhibitor of fatty acid synthase. ${ }^{26}$

The negligible activity of FASN in normal tissues and its seemingly essential role in tumours, begs the question 'why FASN activity is essential for tumour cell survival?' Several hypotheses can be entertained.

(1) FASN has been shown to maintain membrane biogenesis for the endoplasmatic reticulum (ER), which is specialized in the synthesis and transport of secretory and membrane proteins, as well as of phospholipids. A recent study showed that FASN inhibitors induce ER stress in tumour cells. ${ }^{32}$ ER stress is a coordinated cellular response to alterations of ER homeostasis that results in the activation of an adaptive survival response. The same study showed that FASN inhibitors cooperate with the ER stress-inducer thapsigargin to cause cell death. It has thus been proposed that FASN overexpression in tumour cells may be necessary to preserve ER function. 


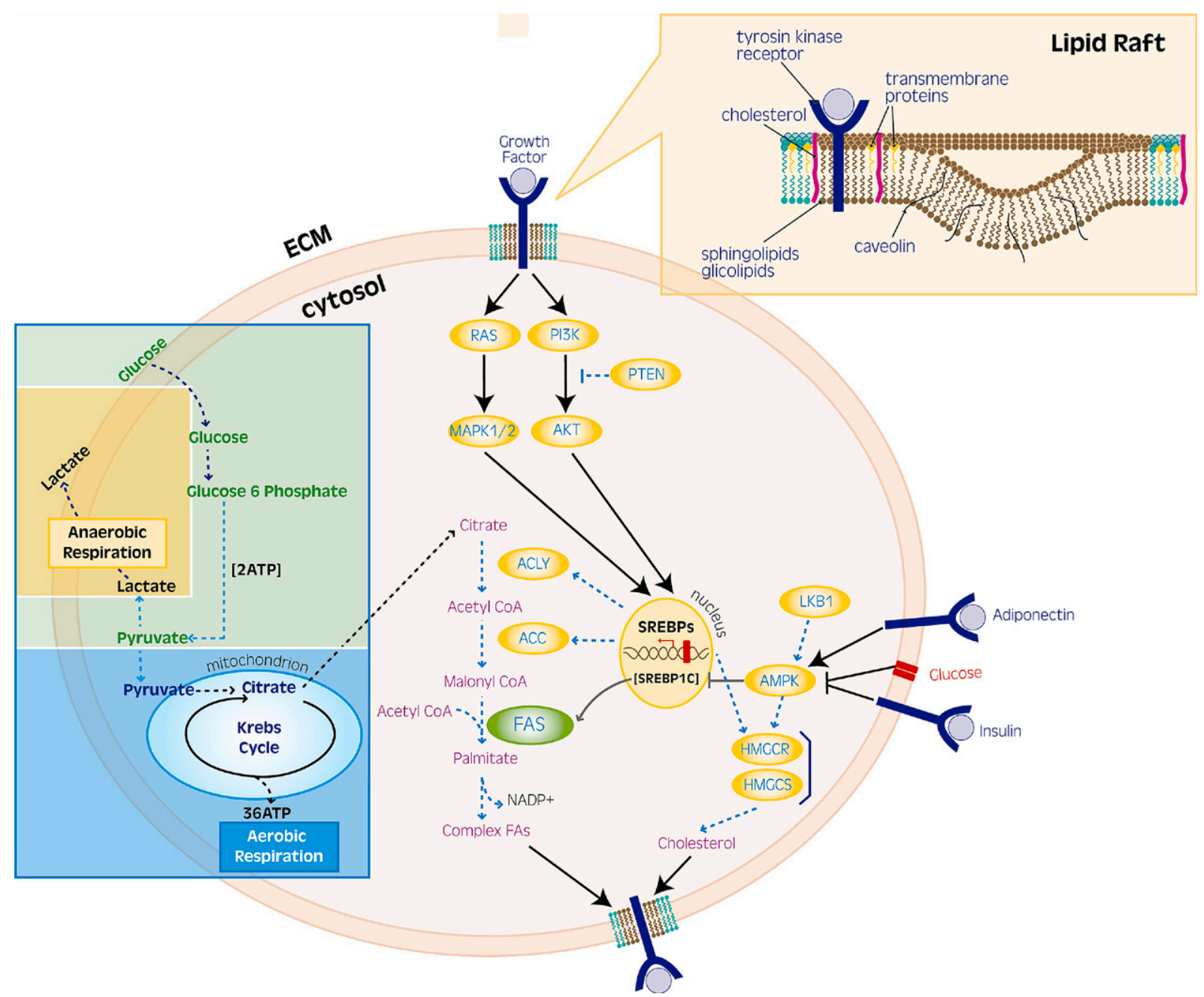

Figure 1 Lipogenesis from glucose metabolism and lipid rafts genesis. The pathway involving the conversion of glucose to fatty acids (FAs) begins with citrate metabolism. In healthy cells, citrate is fully oxidized into the Krebs cycle to generate ATP. In tumour cells, citrate is preferentially transported to the cytoplasm, where it is converted into acetyl-CoA, which constitutes the building block of FAs. Complex FAs, in turn, tend to partition into detergent-resistant membrane microdomains called lipid rafts, which have a role either in cell membranes genesis and in signalling molecules regulation. Key molecule in this metabolic switch-essential for tumour cells survival-is FASN, the enzyme that catalyzes the first step of de novo synthesis of FAs.

(2) Detergent-resistant membrane microdomains may be altered by increased lipid synthesis, thus affecting signal transduction. Most newly synthesized lipids are phospholipids enriched in saturated and monounsaturated fatty acyl chains. These phospholipids, together with cholesterol, tend to partition into detergentresistant membrane microdomains called lipid rafts (Figure 1$)^{33}$ Raft microdomains are also rich in proteins and serve as membrane platforms for signal transduction in various cellular pathways, including the protein kinase $\mathrm{C}$ (PKC), mitogen-activated protein kinase (MAPK), and PI3K pathways. Lipid rafts mobilize and, thus, modulate the activity of the membranous second messangers of these pathways. ${ }^{34}$ The first evidence linking lipid rafts to PCa comes from the identification of the raft-associated protein caveolin1 (Cav-1) as a marker for aggressive PCa. ${ }^{35}$ In a recent study aimed at investigating Cav-1 in the onset and progression of PCa, Cav-1 null mice were interbred with TRAMP mice. Inactivation of Cav-1 resulted in significant reductions in prostate tumour size and occurrence of metastases. ${ }^{36}$ This effect appears to be mediated, at least in part, by FASN, which is downstream of Cav-1. ${ }^{37}$ Signal transduction through rafts in cancer cells may be also be caveolin-independent. Zhuang et al. demonstrated that, in caveolin-negative human PCa cells LNCaP, the EGFR-mediated activation of the PI3K/Akt signaling pathway is downregulated after treatment with the raft-disrupting agent filipin, and is totally recovered by repletion of the membrane with cholesterol. Treatment with filipin alone resulted in cell apoptosis to a similar extent as the PI3K inhibitor, suggesting that the raft microdomains are prominent mediators of survival in PCa cells. ${ }^{38}$ Given the abundance of complex fatty acids in liquid-ordered lipid microdomains, FASN-driven increase in the proportion of membrane phospholipids may result in a modified organization of signalling proteins on the membrane, and thus in a deregulation of several processes (e.g. cell growth and survival).

(3) PCa cells overexpressing FASN may rely on palmitate metabolism to maintain their malignant phenotype. Many of the effects of FASN inhibition such as apoptosis, reactive oxygen 
species (ROS) production and in vivo growth inhibition, have been shown to be at least partially rescued by palmitate. ${ }^{39}$ Palmitate, the principal product of FASN activity, is a signal for cellular membrane localization and activation for many proteins. Palmitoylation of oncogenic proteins like Ras and Wnt is an essential posttranslational modification critical for cancer progression. ${ }^{40}$ In prostate cells overexpressing FASN, global protein palmitoylation profiles reveal unique patterns compared to isogenic cells without FASN overexpression (Migita, Loda et al., unpublished), suggesting an important mechanism through which high levels of FASN, or possibly even exogenous fatty acids (such as those derived from the diet) can influence signalling in PCa cells.

(4) Tumour cells consistently rely on anaerobic pathways: this limits their respiratory chain and, in turn, their oxidizing power. Cancer cells may therefore favour synthesis and chain elongation of fatty acids because they supply oxidizing power for key oxidative steps, to improve the cellular redox balance despite hypoxic conditions. ${ }^{41}$ This would accomplish a reduction in lactic acidosis and the synthesis of fatty acids to be utilized as structural lipids or as an energy source.

It is unclear which of the mechanisms mentioned above, by no means mutually exclusive, is principally responsible for the oncogenic properties of FASN.

FASN regulation: the sterol regulatory element binding proteins (SREBPs) are key players in the transcriptional regulation of lipogenic enzymes. FASN transcription is modulated by the androgen-dependent SREBP1c in the androgen-dependent and ligand-independent (castration-resistant) state. The putative role of FASN in the progression of PCa toward androgen independence is unknown. In the CWR22 model of PCa, FASN protein expression, tumour size and proliferation rate decrease after castration and return to normal with androgen replenishment. ${ }^{42}$ However PCa in castrated mice, which relapsed after a long latency, displayed high levels of FASN protein, as well as tumour growth arrest by FASN inhibitors has been also achieved in some androgen-independent prostate cancer xenografts. ${ }^{39}$ These data suggest an androgen-independent regulation of FASN that is confirmed also in gene expression profile analysis of human prostate cancers. Thus, FASN represents an attractive drug target in androgen-dependent and -independent disease.

A substantial number of PCa patients exhibit discordance between FASN mRNA and protein levels, suggesting posttranscriptional regulatory mechanisms. ${ }^{43}$ FASN protein levels are modulated via de-ubiquitination by the isopeptidase USP2a. USP2a is a pre-proteosomal, androgen-regulated de-ubiquitinating enzyme overexpressed in $\sim 40 \%$ of $\mathrm{PCa}^{44}$ that plays a key part in PCa cell survival through stabilization of FASN protein. ${ }^{45}$ RNA interference of USP2a results in apoptosis of PCa cells, which is rescued by FASN overexpression, suggesting that FASN downregulation can also be achieved by targeting USP2a in PCa.

FASN and the metabolic syndrome (MS): MS is a pathological condition characterized primarily by insulin resistance, and is associated with a high risk for cardiovascular disease and death. The overabundance of circulating fatty acids is the major contributor to the development of insulin resistance in diabetic patients. ${ }^{46}$ In large epidemiologic studies, obesity and the MS have been related to risk of advanced or lethal PCa. ${ }^{47}$ Among men diagnosed with PCa, weight gain before and obesity at the time of cancer diagnosis are associated with an increased probability of PSA failure after prostatectomy.

Lack of AMPK (5'AMP-activated protein kinase) activity is associated with the MS. ${ }^{46}$ AMPK acts as an energy sensor or 'fuel gauge' with the ultimate aim of enhancing ATP generation in low fuel (high AMP/ATP) conditions and plays a crucial part in systemic energy balance by integrating nutritional and hormonal signals in peripheral tissues and also in the hypothalamus. ${ }^{26}$ AMPK modulates FASN expression (by regulating the transcriptional factor SREBP1c) and FASN activity (by phosphorylating and inhibiting acetyl-CoA carboxylase (ACC), which catalyzes the formation of malonyl CoA, the rate-limiting step in fatty acid synthesis, Figure 1). ${ }^{26}$ AMPK stimulation is capable of inhibiting FASN activity in vitro despite constitutive FASN expression in PCa cells.

Therefore, one could speculate that prolonged inactivation of AMPK may contribute to abnormal cellular metabolism and tumour cell transformation/survival through an increase in the availability of circulating fatty acids or the deregulated activity of FASN itself, thus linking predisposing dietary factors and intrinsic alterations of metabolic enzymes, such as FASN. Further evidence is necessary to substantiate the hypothesis that chronic inactivation of AMPK results in unchecked activity of FASN.

FASN: structure-function relationship: The FDA-approved drug orlistat can induce apoptosis in FAS-overexpressing prostate tumours by inhibiting the thioesterase domain of FAS. ${ }^{39}$ The structure of the thioesterase domain of FAS has been resolved. ${ }^{48}$ The structure of $\mathrm{FAS}^{26}$, USP2 ${ }^{49}$ and $\mathrm{AMPK}^{50}$ have also been defined. This will allow for the design of more specific drugs that will affect metabolic pathways crucial to the survival of PCa cells.

\section{Conclusions}

In this review, the authors suggested that interfering with lipid metabolism has detrimental effects on prostate tumour cell survival. Studies are under way to determine the precise mechanisms responsible for tumour initiation and maintenance by altered metabolic pathways.

From a broader perspective, the authors would like to underscore the necessity of more thorough investigations of the metabolic alterations in cancer, and speculate that combining metabolic profiling with genome-wide analyses will drive new understanding in PCa biology and subsequently treatment.

\section{REFERENCES}

1 Walczak JR, Carducci MA. Prostate cancer: a practical approach to current management of recurrent disease. Mayo Clin Proc 2007; 82: 243-9.

2 Parkin DM, Bray F, Ferlay J, Pisani P. Global cancer statistics, 2002. CA Cancer J Clin 2005; 55: 74-108.

3 Wright ME, Chang SC, Schatzkin A, et al. Prospective study of adiposity and weight change in relation to prostate cancer incidence and mortality. Cancer 2007. 
4 Loeb S, Catalona WJ. Prostate-specific antigen in clinical practice. Cancer Lett 2007; 249: 30-39.

5 Thompson IM, Pauler DK, Goodman PJ, et al. Prevalence of prostate cancer among men with a prostate-specific antigen level $<$ or $=4.0$ ng per milliliter. $N$ Engl J Med 2004; 350: 2239-46.

6 D’Amico AV, Whittington R, Malkowicz SB, et al. Biochemical outcome after radical prostatectomy, external beam radiation therapy, or interstitial radiation therapy for clinically localized prostate cancer. JAMA 1998; 280: 969-74.

7 Cooperberg MR, Lubeck DP, Meng MV, Mehta SS, Carroll PR. The changing face of low-risk prostate cancer: trends in clinical presentation and primary management. J Clin Oncol 2004; 22: 2141-9.

8 Tannock IF, de Wit R, Berry WR, et al. Docetaxel plus prednisone or mitoxantrone plus prednisone for advanced prostate cancer. $N$ Engl J Med 2004; 351: 1502-12.

9 Bostwick DG, Shan A, Qian J, et al. Independent origin of multiple foci of prostatic intraepithelial neoplasia: comparison with matched foci of prostate carcinoma. Cancer 1998; 83: 1995-2002.

10 DeMarzo AM, Nelson WG, Isaacs WB, Epstein JI. Pathological and molecular aspects of prostate cancer. Lancet 2003; 361: 955-64.

11 Freedman ML, Haiman CA, Patterson N, et al. Admixture mapping identifies $8 \mathrm{q} 24$ as a prostate cancer risk locus in African-American men. Proc Nat Acad Sci USA 2006; 103: 14068-73.

12 Amundadottir LT, Sulem P, Gudmundsson J, et al. A common variant associated with prostate cancer in European and African populations. Nat Genet 2006; 38: 652-8.

13 Peehl DM. Primary cell cultures as models of prostate cancer development. Endocr Relat Cancer 2005; 12: 19-47.

14 Bradford TJ, Tomlins SA, Wang X, Chinnaiyan AM. Molecular markers of prostate cancer. Urol Oncol 2006; 24: 538-51.

15 Yu YP, Landsittel D, Jing L, et al. Gene expression alterations in prostate cancer predicting tumor aggression and preceding development of malignancy. J Clin Oncol 2004; 22: 2790-9.

16 Roy-Burman P, Wu H, Powell WC, Hagenkord J, Cohen MB. Genetically defined mouse models that mimic natural aspects of human prostate cancer development. Endocr Relat Cancer 2004; 11: 225-54.

17 Majumder PK, Yeh JJ, George DJ, et al. Prostate intraepithelial neoplasia induced by prostate restricted Akt activation: the MPAKT model. Proc Nat Acad Sci USA 2003; 100: 7841-6.

18 Tomlins SA, Rhodes DR, Perner S, et al. Recurrent fusion of TMPRSS2 and ETS transcription factor genes in prostate cancer. Science 2005; 310: 644-8.

19 Wang J, Cai Y, Ren C, Ittmann M. Expression of variant TMPRSS2/ ERG fusion messenger RNAs is associated with aggressive prostate cancer. Cancer Res 2006; 66: 8347-51.

20 Li LC, Carroll PR, Dahiya R. Epigenetic changes in prostate cancer: implication for diagnosis and treatment. J Natl Cancer Inst 2005; 97: 103-15.

21 De Marzo AM, DeWeese TL, Platz EA, et al. Pathological and molecular mechanisms of prostate carcinogenesis: implications for diagnosis, detection, prevention, and treatment. J Cell Biochem 2004; 91: 459-77.

22 Henrique R, Jeronimo C. Molecular detection of prostate cancer: a role for GSTP1 hypermethylation. Eur Urol 2004; 46: 660-9 (discussion: 669).

23 Warburg O. On the origin of cancer cells. Science 1956; 123: 309-14.

24 Costello LC, Franklin RB. 'Why do tumour cells glycolyse?': from glycolysis through citrate to lipogenesis. Mol Cell Biochem 2005; 280: $1-8$.
25 Kuhajda FP. Fatty-acid synthase and human cancer: new perspectives on its role in tumor biology. Nutrition 2000; 16: 202-208.

26 Menendez JA, Lupu R. Fatty acid synthase and the lipogenic phenotype in cancer pathogenesis. Nat Rev Cancer 2007; 7: 763-77.

27 Kuhajda FP, Pizer ES, Li JN, Mani NS, Frehywot GL, Townsend CA. Synthesis and antitumor activity of an inhibitor of fatty acid synthase. Proc Nat Acad Sci USA 2000; 97: 3450-4.

28 Rossi S, Graner E, Febbo P, et al. Fatty acid synthase expression defines distinct molecular signatures in prostate cancer. Mol Cancer Res 2003; 1: 707-15.

29 Baron A, Migita T, Tang D, Loda M. Fatty acid synthase: a metabolic oncogene in prostate cancer? J Cell Biochem 2004; 91: 47-53.

30 Pizer ES, Pflug BR, Bova GS, Han WF, Udan MS, Nelson JB. Increased fatty acid synthase as a therapeutic target in androgen- independent prostate cancer progression. Prostate 2001; 47: 102-10.

31 Pflug BR, Pecher SM, Brink AW, Nelson JB, Foster BA. Increased fatty acid synthase expression and activity during progression of prostate cancer in the TRAMP model. Prostate 2003; 57: 245-54.

32 Little JL, Wheeler FB, Fels DR, Koumenis C, Kridel SJ. Inhibition of fatty acid synthase induces endoplasmic reticulum stress in tumor cells. Cancer Res 2007; 67: 1262-9.

33 Swinnen JV, Van Veldhoven PP, Timmermans L, et al. Fatty acid synthase drives the synthesis of phospholipids partitioning into detergent-resistant membrane microdomains. Biochem Biophys Res Commun 2003; 302: 898-903.

34 Freeman MR, Solomon KR. Cholesterol and prostate cancer. J Cell Biochem 2004; 91: 54-69.

35 Yang G, Truong LD, Wheeler TM, Thompson TC. Caveolin-1 expression in clinically confined human prostate cancer: a novel prognostic marker. Cancer Res 1999; 59: 5719-23.

36 Williams TM, Hassan GS, Li J, et al. Caveolin-1 promotes tumor progression in an autochthonous mouse model of prostate cancer: genetic ablation of Cav-1 delays advanced prostate tumor development in tramp mice. J Biol Chem 2005; 280: 25134-145.

37 Di Vizio D, Sotgia F, Williams TM, et al. Caveolin-1 is required for the upregulation of fatty acid synthase (FASN), a tumor promoter, during prostate cancer progression. Cancer Biol Ther 2007; 6: 1263-8.

38 Zhuang L, Lin J, Lu ML, Solomon KR, Freeman MR. Cholesterol-rich lipid rafts mediate akt-regulated survival in prostate cancer cells. Cancer Res 2002; 62: 2227-31.

39 Kridel SJ, Axelrod F, Rozenkrantz N, Smith JW. Orlistat is a novel inhibitor of fatty acid synthase with antitumor activity. Cancer Res 2004; 64: 2070-5.

40 Kato K, Der CJ, Buss JE. Prenoids and palmitate: lipids that control the biological activity of Ras proteins. Semin Cancer Biol 1992; 3: 179-88.

41 Hochachka PW, Rupert JL, Goldenberg L, Gleave M, Kozlowski P. Going malignant: the hypoxia-cancer connection in the prostate. Bioessays 2002; 24: 749-57.

42 Myers RB, Oelschlager DK, Weiss HL, Frost AR, Grizzle WE. Fatty acid synthase: an early molecular marker of progression of prostatic adenocarcinoma to androgen independence. J Urol 2001; 165: 1027-32.

43 Rossi S, Graner E, Febbo P, et al. Fatty acid synthase expression defines distinct molecular signatures in prostate cancer. Mol Cancer Res 2003; 1: 707-15.

44 Priolo C, Tang D, Brahamandan M, et al. The isopeptidase USP2a protects human prostate cancer from apoptosis. Cancer Res 2006; 66: $8625-32$. 
45 Graner E, Tang D, Rossi S, et al. The isopeptidase USP2a regulates the stability of fatty acid synthase in prostate cancer. Cancer Cell 2004; 5: 253-61.

46 Ruderman N, Prentki M. AMP kinase and malonyl-CoA: targets for therapy of the metabolic syndrome. Nat Rev Drug Discov 2004; 3: 340-51.

47 Laukkanen JA, Laaksonen DE, Niskanen L, Pukkala E, Hakkarainen A, Salonen JT. Metabolic syndrome and the risk of prostate cancer in Finnish men: a population-based study. Cancer Epidemiol Biomarkers Prev 2004; 13: 1646-50.

48 Chakravarty B, Gu Z, Chirala SS, Wakil SJ, Quiocho FA. Human fatty acid synthase: structure and substrate selectivity of the thioesterase domain. P. Proc Nat Acad Sci USA 2004; 101: 15567-72.

49 Renatus M, Parrado SG, D’Arcy A, et al. Structural basis of ubiquitin recognition by the deubiquitinating protease USP2. Structure 2006; 14: 1293-302.

50 Townley R, Shapiro L. Crystal structures of the adenylate sensor from fission yeast AMP-activated protein kinase. Science 2007; 315: 1726-9.

\section{Practice points}

- Novel molecular biomarkers have the potential to improve risk stratification among patients with prostate cancer ( $\mathrm{PCa}$ ) and allow for better decision-making about treatment.

- Candidate high-risk genes that are potentially associated with hereditary PCa are RNAse L and MSR1; many sporadic $\mathrm{PCas}$, characterized by heterogeneous patterns of oncogene activation, show the commonest chromosomal rearrangement yet identified in human solid tumours, resulting in the fusion of TMPRSS2 (21q22.2) with ERG (21q22.3) or ETV1 (7p21.2).

- Upregulation of fatty acid synthase (FASN), the key metabolic enzyme that is responsible for the terminal catalytic step in de novo synthesis of fatty acids (FAs), represents a common characteristic in human malignancies.
- FASN seems to play a crucial role in PCa by conferring growth and survival advantages to cancer cells, maintaining membrane biogenesis and membranous signalling proteins regulation, and providing protein palmitoylation, which is a post-translational modification critical in cancer progression.

- The metabolic syndrome (MS), which is a pathological condition characterized by primarily insulin resistance and associated with the overabundance of circulating fatty acids, has been related to risk of advanced PCa. Prolonged inactivation of AMPK in patients with PCa may contribute to abnormal cellular metabolism and tumour cell transformation/ survival by promoting the unchecked activity of FASN.

- Tumour exacerbated lipogenesis might represent a potential marker in tumour diagnosis (because it emerges early in carcinogenesis) and a target in treatment of advanced carcinomas.

\section{Acknowledgments}

We thank members of Loda laboratory for fruitful discussions, particularly Emanuele Palesandolo, Carmen Priolo, Stephen Finn and Giorgia Zadra; Sonal Jhaveri-Schneider for critical review of the manuscript; Marleen Marino and Jane Hayward for graphic assistance.

This work was supported by the Gelb Center for genitourinary oncology and by grants from the NIH (Specialized Programs of Research Excellence 5P50CA90381, P01 CA089021) and the Prostate Cancer Foundation (Massimo Loda). ML is a Novartis investigator. 